\title{
Enhanced Photoinduced Desorption from Metal Nanoparticles by Photoexcitation of Confined Hot Electrons Using Femtosecond Laser Pulses
}

\author{
Ki Hyun Kim, Kazuo Watanabe, ${ }^{*}$ Daniel Mulugeta, Hans-Joachim Freund, and Dietrich Menzel ${ }^{\dagger, *}$ \\ Fritz-Haber-Institut der Max-Planck-Gesellschaft, Faradayweg 4-6, 14195 Berlin, Germany
}

(Received 14 October 2010; published 21 July 2011)

\begin{abstract}
Strong fluence dependence of photodesorption cross sections is observed in femtosecond laser photodesorption of $\mathrm{NO}$ from $(\mathrm{NO})_{2}$ on silver nanoparticles, in contrast to femtosecond photodesorption on bulk metals. The time scale of excitation buildup is found to be equal or less than the pulse duration of $\sim 100 \mathrm{fs}$; NO translational energies are independent of fluence and pulse duration. We propose a nanoparticle-specific nonlinear mechanism in which, due to confinement, strongly nonthermal hot-electron distributions are maintained during the femtosecond pulses, enhancing the normal desorption pathway.
\end{abstract}

DOI: 10.1103/PhysRevLett.107.047401

PACS numbers: 78.67.Bf, 68.43.Tj, 73.20.Mf, 82.53.St

Metal nanoparticles (NPs) show remarkable changes of electronic and optical properties vs the bulk metals [1]. In particular, confinement of excitations in the NPs and plasmon excitation can influence photochemistry proceeding on them [2]. Strong plasmon enhancement and particle size dependence were observed for photoreactions of NO dimers on silver NPs compared to bulk $\mathrm{Ag}(111)[3,4]$. This work used nanosecond (ns) excitation, which leads to linear response of yields to fluence, so that cross sections are independent of fluence. In this regime the final state energy distributions are constant and compatible [3,4] with a desorption mechanism via a transient negative ion (TNI) state [5].

Photodesorption on surfaces of bulk single crystals has long been studied in detail $[5,6]$. Besides the range of linear behavior, nonlinear desorption processes induced by femtosecond (fs) laser pulses have been seen which enhance the yield, and mechanisms have been established [7-9]. The different response of NPs to excitations [1,2] suggests that fs excitation could act differently on them. The only published fs desorption data for NPs, for $\mathrm{H}_{2} \mathrm{O}$ desorption from $\mathrm{Ag}$ NPs [10], were interpreted as due to transient heating of the NPs. We present here an investigation of the photodesorption from NPs by fs excitation in our welldefined test system, photodesorbed NO from adsorbed $(\mathrm{NO})_{2}$ on supported Ag NPs [3]. We find a strong fluence dependence of cross sections for fs excitation implying nonlinear effects on the yield; with plasmon enhancement this occurs at very low fluences. Constant NO translational energies suggest that no change of the desorption dynamics occurs. The relevant excitation lifetime indicated by twopulse correlation measurements is found to be very short. These results cannot be explained by the mentioned nonlinear desorption mechanisms for single-crystal surfaces [7-9], as these would call for longer excitation lifetimes and varying translational energies, nor by thermal effects. We propose a mechanism specific to NPs which depends on the reexcitation of confined hot electrons in the NPs during a single laser pulse. While we here explain fs photodesorption in a particular system, we believe that this mechanism can generally work in fs photodesorption from NPs and that its main ingredient, nonlinear reexcitation within the NPs, might even have implications for other fast electronic processes on NPs, such as two-photon photoemission or nonlinear interaction of plasmons, which necessitate the use of fs pulses.

The experiments were done in a two-chamber ultrahigh vacuum system, for sample preparation and characterization and for measurement. In the latter chamber the sample can be irradiated by ns $(\sim 5 \mathrm{~ns}, 3.5 \mathrm{eV})$ or fs $(\sim 100 \mathrm{fs}$, $3.1 \mathrm{eV}$ ) laser pulses. Ag NPs (mean diameter $8 \mathrm{~nm}$ ) on double layers of $\mathrm{Al}_{2} \mathrm{O}_{3}$ on $\mathrm{NiAl}(110)$ [11] were prepared; identical measurements on an $\operatorname{Ag}(111)$ crystal were done in situ for comparison. Saturated (NO) $)_{2}$ monolayers [3] were produced at $75 \mathrm{~K}$ on the NPs (no NO is adsorbed on the oxide as proved earlier $[3,4]$ ) and cooled to $30 \mathrm{~K}$ for irradiation. Desorbing NO molecules are detected in a mass-selected time-of-flight (MS-TOF) [3] setup. Desorption yields (from the signal strength), cross sections (from the logarithmic signal decay normalized to fluence $[3,5,6]$ ), and translational energies (from the TOF spectra) have been determined. Thermal effects can be safely excluded [12].

Figure 1 shows the irradiation-induced decay of the desorption signal of neutral NO vs total number of impinged photons, comparing $\mathrm{ns}(3.5 \mathrm{eV}, p$-polarized) and fs (3.1 eV, $p$-polarized) irradiation of $(\mathrm{NO})_{2}$ on $\mathrm{Ag}(111)$ [Fig. 1(a)] and on Ag NPs [Fig. 1(b)] [the $(1,0)$ mode of the Mie plasmon of these Ag NPs is excited only by $p$-polarized light ( $p$-polarized) $[3,4]]$. From such data the photodesorption cross sections (PCSs) can be derived $[3,5,6]$. On $\mathrm{Ag}(111)$ the PCSs for ns and fs excitation are about the same up to $2 \mathrm{~mJ} / \mathrm{cm}^{2}$ [Figs. 1 and 2(a)], while on the Ag NPs fs pulses lead to a strong enhancement (sixfold even at $\sim 0.3 \mathrm{~mJ} / \mathrm{cm}^{2}$ ). The energy of $3.1 \mathrm{eV}$ is away from the plasmon maximum (intensity about $20 \%$ of that of the maximum at $3.5 \mathrm{eV}[13,14])$, so that the PCS enhancements are even larger than seen in Fig. 2(a). The true ratios 


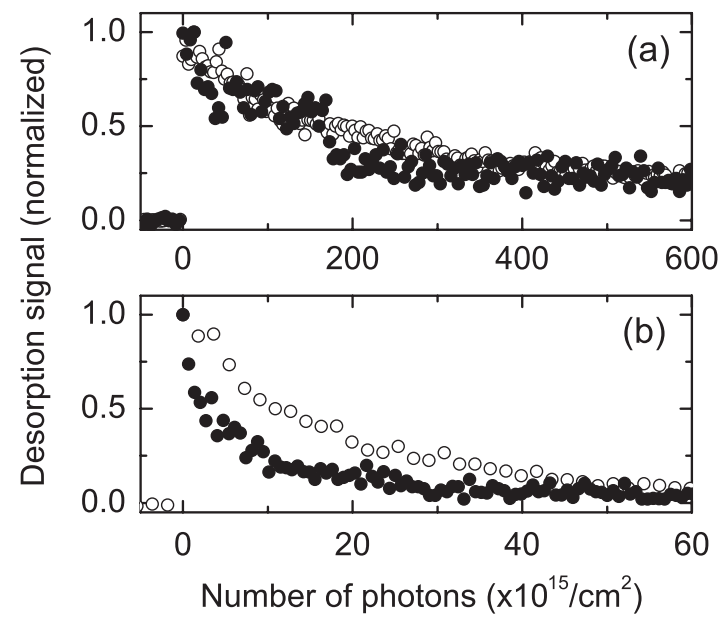

FIG. 1. Irradiation-induced decay of NO desorption signal as a function of the accumulated number of arrived photons in successive shots, for ns (open symbols) and fs (filled symbols) irradiation (a) on $\mathrm{Ag}(111) \quad(3.5 \mathrm{eV} \quad p$-polarized, fluence $\left.2 \mathrm{~mJ} / \mathrm{cm}^{2}\right)$ and (b) on Ag NPs (8 nm mean diameter; $3.5 \mathrm{eV}$ $p$-polarized, fluence $1 \mathrm{~mJ} / \mathrm{cm}^{2}$ for $\mathrm{ns} ; 3.1 \mathrm{eV} p$-polarized, $0.34 \mathrm{~mJ} / \mathrm{cm}^{2}$ for fs pulses). Resulting cross sections are 3.9 , $3.5,59$, and $320 \times 10^{-18} \mathrm{~cm}^{2}$, respectively.

are probably even larger, because the first fs laser pulse reduces the signal by about $30 \%$, so that subsequent pulses probe areas in the wings of the laser intensity profile. Our PCS determination then yields a lower bound.

Indeed, the fluence dependence of the fs desorption yield is distinctly nonlinear [roughly to the third power up to $\sim 0.2 \mathrm{~mJ} / \mathrm{cm}^{2}$ on ( $p$-polarized), with weaker dependence at higher fluence due to the mentioned depletion effect; see
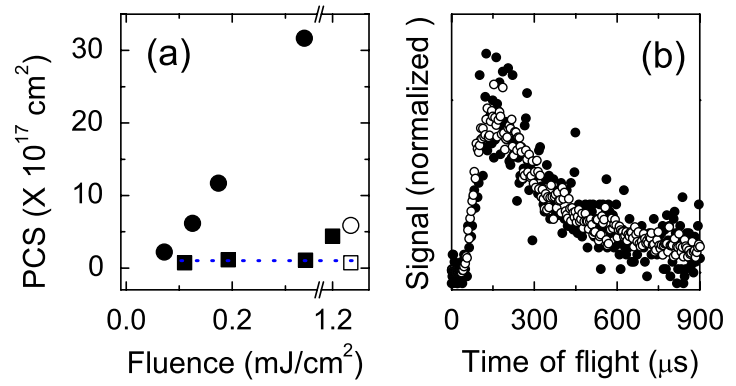

FIG. 2 (color online). (a) PCS from Ag NPs as a function of fluence for fs and ns pulses. Filled circles: $3.1 \mathrm{eV} p$-polarized fs pulses at low fluences. As discussed in the text, the results show lower bounds for the increase of cross sections. Filled squares: $3.1 \mathrm{eV} \mathrm{s}$-polarized fs pulses, constant at low and enhanced at much higher fluences. Open square: $3.5 \mathrm{eV} s$-polarized ns pulses $\left(2 \mathrm{~mJ} / \mathrm{cm}^{2}\right)$; open circle: the same, $p$-polarized $\left(1 \mathrm{~mJ} / \mathrm{cm}^{2}\right)$. The dotted line is a guide for the eye, indicating the constancy of cross sections for fs $s$ polarization at low fluences, agreeing with ns $s$ polarization. (b) Comparison of the time-of-flight spectra, indicative of the translational energy distribution, of desorbing NO from Ag NPs via ns (open circles) and fs (filled circles, $0.4 \mathrm{~mJ} / \mathrm{cm}^{2}, p$-polarized) excitation. The much smaller signals in the fs case lead to the large scatter. Nevertheless, it is clear that the translational energies are unchanged (mean energy $\sim 700 \mathrm{~K}$ ).
[15]]. Consistently, the PCS [Fig. 2(a)] strongly increases with fluence, as expected for a nonlinear yield response. We stress again that linear yield translates into constant cross sections, and any increase of cross sections with fluence indicates nonlinear effects in the yield. So, fs excitation of NPs at the plasmon frequency leads to strong nonlinearity of the desorption yields at low fluences, even though the depletion effect makes an exact determination of the nonlinear exponent impossible. Without plasmon enhancement ( $s$-polarized light), nonlinear yields are observed as well, but about 10 times higher fluences are needed for noticeable PCS enhancement by fs excitation. On $\operatorname{Ag}(111)$ even at more than 10 times higher fluences (up to about $2 \mathrm{~mJ} / \mathrm{cm}^{2}$ ), no trace of yield nonlinearity (or cross section increases) is observed, in agreement with Fig. 1(a). Previous nonlinear desorption on surfaces of bulk material necessitated fluences $>1 \mathrm{~mJ} / \mathrm{cm}^{2}[8,9]$.

Despite these strong efficiency changes, the translational energies of NO desorbed by ns and fs excitation from NPs, investigated by MS-TOF, were found not to change [see Fig. 2(b)], suggesting unchanged desorption dynamics. The data can be represented by shifted MaxwellBoltzmann distributions [3] corresponding to a mean energy of $\sim 700 \mathrm{~K}$.

The yield nonlinearity for the NPs might be expected to be due to one of the nonlinear mechanisms of desorption by fs laser irradiation, desorption induced by multiple electronic transitions (DIMET) $[7,8,16]$, or frictional energy transfer $[8,9,16]$. These mechanisms, well documented for surfaces of compact materials, both work by accumulation of vibrational energy of the adsorbate up to desorption, due to repeated electronic excitation of the same adsorbate during the lifetime of its excited nuclear motion. For DIMET this happens directly, in a few large energy transfers; for frictional coupling many small increments are transferred from the thermalized hot electrons. Both paths can lead to desorption under conditions of very low desorption probability for a single excitation. The lifetimes of vibrational excitations of adsorbates are in the range of many picoseconds (ps) [8,17]; the lifetime of thermalized hot electrons is limited by electron-phonon coupling to a similar range [8]. Two-pulse correlation measurements $[8,18,19]$ for the desorption yield should tell whether one of these mechanisms could apply here. Figure 3 shows the result of such a measurement where the desorption signal for the first shot pair is plotted as a function of pulse delay. Clearly, nonlinear enhancement is seen at short delays (i.e., delays $<200 \mathrm{fs}$ ), but no contribution from longer lifetimes exists which could be ascribed to vibrational lifetimes or long-living thermalized hot electrons, as required for DIMET or frictional energy transfer. Two-pulse correlation of the total electron current from the sample, here due to two-photon photoemission, gave the same result as Fig. 3. While narrow two-pulse correlations do not necessarily exclude contributions from longer-living processes with low yield [20], the high rates observed here exclude such a possibility. 


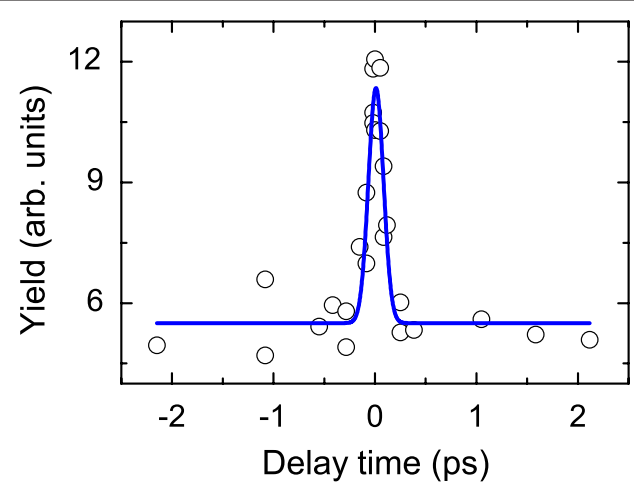

FIG. 3 (color online). Two-pulse correlation measurements at fluences of $0.2 \mathrm{~mJ} /$ pulse. Despite the scatter, it is obvious that the nonlinear enhancement is confined to the pulse duration and does not extend into the ps range. The solid line is a Gaussian distribution best fit.

These results exclude the applicability of both established nonlinear desorption mechanisms and indicate that the fs enhancement seen is governed by electron dynamics within the NPs. The unchanged translational energies [Fig. 2(b)] support this conclusion, since quite different and fluence-dependent translational energies are expected for both DIMET and frictional transfer [21]. This shows that while nonlinear buildup of excitations does proceed with fs excitation on NPs even at very low fluences, the individual desorption event is the same as for ns excitation and even on $\operatorname{Ag}(111)$; i.e., the TNI mechanism via shortlived negative adsorbate ions produced by transfer of hot electrons of sufficient energy prevails. Only the attempt frequency of these excitation events is increased by the high temporal density of energetic hot electrons in the NPs. We propose, therefore, a nonlinear buildup of sufficiently energetic hot electrons by multiple excitations in the NPs during each laser pulse as the basic mechanism. The involvement of such athermally hot electrons in the electron dynamics of NPs has been discussed before in a different context [22].

Theoretical calculations [23] as well as our excitation energy-dependent results [3] show that the lowest unoccupied molecular orbital (LUMO) of the (NO) $)_{2}$ adsorbate, which accepts a hot electron to form its TNI, lies at $\sim 2 \mathrm{eV}$ above the Fermi level $\left(E_{F}\right)$. Absorption of 3.1 or $3.5 \mathrm{eV}$ excites Ag $s$ electrons and creates athermally hot electrons up to $\sim 3 \mathrm{eV}$ (subsequently "primary hot electrons") in the NP. Using $p$-polarized light enhances this process by the plasmon excitation, which decays within about 5-10 fs, mainly by Landau damping $[1,2,24]$. For $s$ polarization, electron-hole pairs are directly created by photon absorption. The primary, athermally hot electrons also decay very rapidly — on a time scale of 5 to at most $10 \mathrm{fs}[2,22,24]$ - by colliding with ground state electrons below $E_{F}$ and (within a few hundred fs) lead to a thermal distribution of hot electrons. These thermalized hot electrons then cool by electron-phonon interaction (describable by a twotemperature model [25]), on a time scale of some ps
$[8,26]$. For ns excitation we estimate that, even by including plasmon enhancement [3], less than one primary hot electron is formed per ps and NP; it has a small chance (within 5-10 fs) to form a TNI before it loses half of its energy by collision with a ground state electron, so neither can access the LUMO any more [Fig. 4(a)]. This explains the linear behavior. With fs excitation the temporal density of primary hot electrons increases by a factor $>10^{4}$ for the same fluence per pulse, and there are of the order of $10^{3}$ such electrons per NP and pulse (of $\sim 100 \mathrm{fs}$ ). Then during one pulse, while the hot electrons are thermalizing, they are continuously reexcited to energies sufficient to enter the LUMO, due to the confinement of hot electrons in the NP. In contrast, at a single-crystal surface the hot electrons largely escape into the bulk. In addition, now the primary hot electrons can also collide with intermediate or thermalized hot electrons, so that both may receive sufficient energy for the TNI formation [see Figs. 4(b) and 4(c)]. For excitation in the plasmon resonance ( $p$-polarized), even a direct plasmon-hot-electron interaction might exist. This could explain that the ratio of cross sections for $p$ and $s$ polarization exceeds the plasmon enhancement seen in absorption. All these processes nonlinearly increase the number of electrons initiating the TNI sequence which leads to desorption. However, the nuclear dynamics of the evolution of each TNI, i.e., each individual desorption event, remains the same. The main basis for the suggested mechanism is the confinement of the excited electrons in

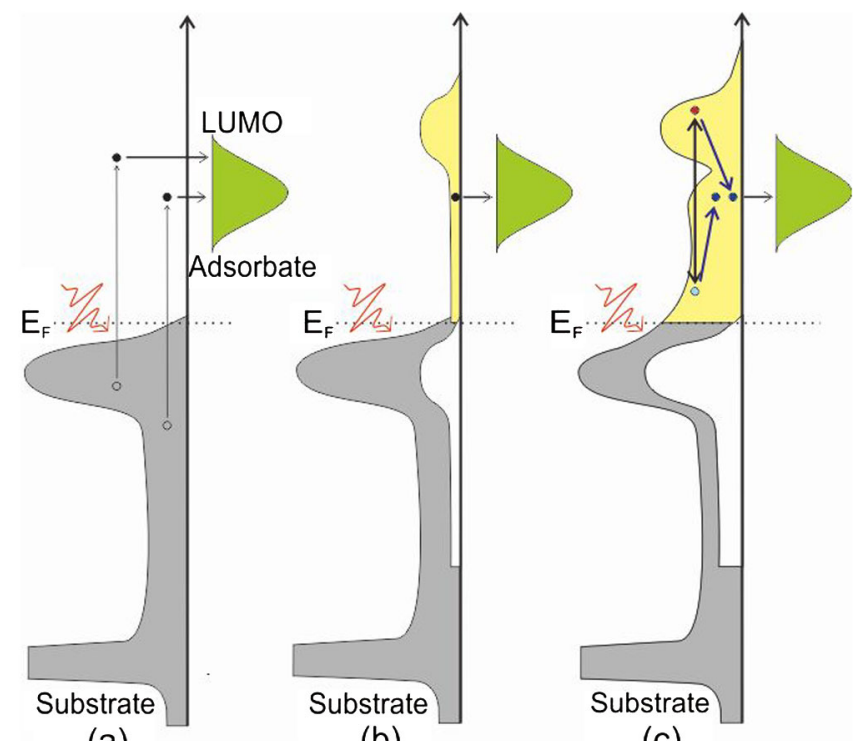

(a)

(b)

(c)

FIG. 4 (color online). Sketch of the suggested new nonlinear excitation mechanism working only in NPs, compared to the linear process. (a) Single desorption attempt via TNI in the linear regime (ns excitation). (b) Strong excitation of primary hot electrons in the early stage (before thermalization sets in) of a fs pulse. (c) Situation at the end of a fs pulse, with high density (exaggerated) of energetic hot electrons created by repumping and collisions between hot electrons, and a thermalized tail of hot electrons. 
the NP which makes them accessible to reexcitation. Therefore, this mechanism is specific to NPs.

In summary, for NO photodesorption from adsorbed $(\mathrm{NO})_{2}$ on Ag NPs we observe a strong increase with photon fluence of desorption cross sections by fs excitation, compared to ns excitation. In the range of the Mie plasmon this sets in at very low fluences; somewhat higher fluences are necessary for $s$ polarization (direct electron-hole creation without plasmon enhancement). No such effect is seen on $\operatorname{Ag}(111)$, where excitations can escape into the bulk. Twopulse correlation measurements show that the involved excitations possess a very short lifetime (of the order of or below the laser pulse width), so that energy accumulation in the adsorbate vibrations by repetitive excitations (direct or by thermalized hot electrons), as operative in the known nonlinear mechanisms DIMET and frictional coupling, cannot be responsible. The translational energies of the desorbing NO remain unchanged compared to linear ns processes, showing the individual desorption event to be the same in the linear and nonlinear ranges. We propose a new desorption mechanism induced by nonlinear accumulation of energetic hot electrons in the NPs, which is based on hot-electron confinement and therefore works only for NPs: buildup and repumping of energetic hot electrons within the NPs by the high temporal and spatial density of excitations. It may be further enhanced by plasmon excitation. Theoretical modeling of the suggested nonequilibrium distributions of the confined hot electrons would be highly desirable. We believe that this mechanism has broader applicability for fs photochemistry on NPs and other fast processes on them.

We thank J. Bdzoch, U. Bovensiepen, Ch. Frischkorn, J. Stähler, and M. Wolf for helpful suggestions and discussions related to the experiments and U. Höfer for valuable discussions and for calling our attention to Ref. [20]. We acknowledge financial support from the Deutsche Forschungsgemeinschaft within priority program SPP1093 (Dynamik von Elektronentransferprozessen an Grenzflächen), the German-Israeli Foundation (Dynamics of Electronic Processes in a Confined Environment), the Fonds der Chemischen Industrie, and the NEDO International Joint Research Grant on Photon and Electron Controlled Surface Processes.

*Present address: Department of Chemistry, Tokyo University of Science, 1-3 Kagurazaka, Shinjuku-ku, Tokyo 162-8601, Japan.

†Corresponding author. dietrich.menzel@ph.tum.de

Also at Physik-Department E20, Technische Universität München, 85748 Garching, Germany.

[1] U. Kreibig and M. Vollmer, Optical Properties of Metal Clusters (Springer, Berlin, 1995).
[2] K. Watanabe, D. Menzel, N. Nilius, and H.-J. Freund, Chem. Rev. 106, 4301 (2006).

[3] D. Mulugeta, K. H. Kim, K. Watanabe, D. Menzel, and H.J. Freund, Phys. Rev. Lett. 101, 146103 (2008).

[4] D. Mulugeta, K. Watanabe, D. Menzel, and H.-J. Freund, J. Chem. Phys. 134, 164702 (2011).

[5] F. M. Zimmermann and W. Ho, Surf. Sci. Rep. 22, 127 (1995).

[6] See, e.g., D. Menzel, Surf. Interface Anal. 38, 1702 (2006), and references therein.

[7] J. A. Misewich, T. F. Heinz, and D. M. Newns, Phys. Rev. Lett. 68, 3737 (1992).

[8] For a recent review, see Ch. Frischkorn and M. Wolf, Chem. Rev. 106, 4207 (2006).

[9] P. Avouris and B. N. J. Persson, J. Phys. Chem. 88, 837 (1984).

[10] S. Kwiet, D. E. Starr, A. Grujic, M. Wolf, and A. Hotzel, Appl. Phys. B 80, 115 (2005).

[11] J. Libuda, F. Winkelmann, M. Bäumer, H.-J. Freund, T. Bertrams, H. Neddermeyer, and K. Müller, Surf. Sci. 318, 61 (1994).

[12] Calculations indicate negligible thermal effects at these low fluences and are corroborated by the fact that $\mathrm{N}_{2} \mathrm{O}$ molecules, which are a by-product of the NO desorption, remain on the surface after irradiation and can be detected by thermal desorption below $120 \mathrm{~K}$. This proves that this temperature has not been surpassed during irradiation.

[13] N. Nilius, N. Ernst, and H.-J. Freund, Phys. Rev. Lett. 84, 3994 (2000).

[14] F. Evers, C. Rakete, K. Watanabe, D. Menzel, and H.-J. Freund, Surf. Sci. 593, 43 (2005).

[15] See Supplemental Material at http://link.aps.org/ supplemental/10.1103/PhysRevLett.107.047401 for fluence dependence of the desorption yield.

[16] M. Brandbyge, P. Hedegard, T. F. Heinz, J. A. Misewich, and D. M. Newns, Phys. Rev. B 52, 6042 (1995).

[17] R. R. Cavanagh, D. S. King, J. C. Stephenson, and T. F. Heinz, J. Phys. Chem. 97, 786 (1993).

[18] F. Budde, T. F. Heinz, M. M. T. Loy, J. A. Misewich, F. Derougemont, and H. Zacharias, Phys. Rev. Lett. 66, 3024 (1991).

[19] F. J. Kao, D. G. Busch, D. Cohen, D. G. Dacosta, and W. Ho, Phys. Rev. Lett. 71, 2094 (1993).

[20] M. Lawrenz, K. Stepan, J. Gudde, and U. Höfer, Phys. Rev. B 80, 075429 (2009).

[21] D. G. Busch, S. W. Gao, R. A. Pelak, M. F. Booth, and W. Ho, Phys. Rev. Lett. 75, 673 (1995).

[22] C. Voisin, D. Christofilos, N. Del Fatti, and F. Vallée, Eur. Phys. J. D 16, 139 (2001).

[23] H. Nakamura and K. Yamashita, J. Chem. Phys. 125, 084708 (2006).

[24] M. Merschdorf, C. Kennerknecht, and W. Pfeiffer, Phys. Rev. B 70, 193401 (2004).

[25] S. I. Anisimov, B. L. Kapeliovich, and T. L. Perelman, Zh. Eksp. Teor. Fiz. 66, 776 (1974).

[26] M. Lisowski, P. A. Loukakos, U. Bovensiepen, J. Stähler, C. Gahl, and M. Wolf, Appl. Phys. A 78, 165 (2004). 